Lecture Notes in Mathematics Vol. 334

ISBN 978-3-540-47010-6 (C) Springer-Verlag Berlin Heidelberg 2008

Fritz Schweiger

\title{
The Metrical Theory of Jacobi-Perron Algorithm
}

\section{Errata}

It was not before 1997 that a serious flaw in the proof of theorem 8.7. was detected by Adriana Berechet. An attempt to save the result can be found in F. Schweiger: Kuzmin's theorem revisited. Ergodic Theory and Dynamical Systems 20 (2000), 557-565. However, the new proof gives a weaker convergence rate.

More information on the Jacobi-Perron algorithm and other multidimensional continued fractions can be found in A. J. Brentjes: Multi-dimensional continued fraction algorithms. Mathematical Centre Tracts 145, Amsterdam: Mathematisch Centrum 1981 and in F. Schweiger: Multidimensional Continued Fractions. Oxford: Oxford University Press 2000. 